\title{
Experimental Satellite Quantum Communications
}

\author{
Giuseppe Vallone, ${ }^{1}$ Davide Bacco, ${ }^{1}$ Daniele Dequal, ${ }^{1}$ Simone Gaiarin, ${ }^{1}$ Vincenza Luceri, ${ }^{2}$ \\ Giuseppe Bianco, ${ }^{3}$ and Paolo Villoresi ${ }^{1, *}$ \\ ${ }^{1}$ Dipartimento di Ingegneria dell'Informazione, Università degli Studi di Padova, Padova 35131, Italy \\ ${ }_{e}^{2}$-GEOS spa, Matera 75100 , Italy \\ ${ }^{3}$ Matera Laser Ranging Observatory, Agenzia Spaziale Italiana, Matera 75100, Italy
}

(Received 13 April 2015; revised manuscript received 26 May 2015; published 20 July 2015)

\begin{abstract}
Quantum communication $(\mathrm{QC})$, namely, the faithful transmission of generic quantum states, is a key ingredient of quantum information science. Here we demonstrate QC with polarization encoding from space to ground by exploiting satellite corner cube retroreflectors as quantum transmitters in orbit and the Matera Laser Ranging Observatory of the Italian Space Agency in Matera, Italy, as a quantum receiver. The quantum bit error ratio (QBER) has been kept steadily low to a level suitable for several quantum information protocols, as the violation of Bell inequalities or quantum key distribution (QKD). Indeed, by taking data from different satellites, we demonstrate an average value of QBER $=4.6 \%$ for a total link duration of $85 \mathrm{~s}$. The mean photon number per pulse $\mu_{\text {sat }}$ leaving the satellites was estimated to be of the order of one. In addition, we propose a fully operational satellite QKD system by exploiting our communication scheme with orbiting retroreflectors equipped with a modulator, a very compact payload. Our scheme paves the way toward the implementation of a QC worldwide network leveraging existing receivers.
\end{abstract}

Introduction.-Quantum information protocols may require short links, as in the case of quantum computing, or very long ones, as for the realization of quantum key distribution (QKD) in which two parties generate a secret key for data encryption [1-6]. Such parties may be connected by optical cables [7-9], or by free-space optical links [10]. However, point-to-point fiber links are limited to a few hundred kilometers due to optical attenuation. Free-space links on ground are similarly limited, due to the curvature of the Earth and to atmospheric attenuation and turbulence. The development of quantum repeaters may extend such limits at the cost of introducing a remarkable complexity. On the other hand, free-space satellite links would allow the realization of a global quantum communication network as fostered in several continental information-and-communicationtechnology roadmaps [11,12]. Indeed, QKD [1-6], quantum teleportation [10], and entanglement swapping [13]—as well as the measurement of Bell inequalities in a relativistic scenario [14] and fundamental tests of quantum physics [15]—require quantum communication (QC) over long distances and, in particular, along satellite links. Moreover, these protocols can be further developed exploiting higherdimensional Hilbert spaces [5,6].

The envisaging and modeling of space QC started a dozen years ago [16-20], but sources of quantum states or quantum state measurement systems suitable for QC have not been placed in orbit yet. Therefore, since 2008, experimental studies of space-to-ground links have simulated a source of coherent pulses attenuated to the single photon level by exploiting laser ranging satellites equipped with corner-cube retroreflectors (CCRs) [21,22]. However, polarization encoded QKD in space requires qubits prepared in different polarization states. Previous studies [21,22] did not experimentally demonstrate either single photon polarization preservation or discrimination between different polarization encoded quantum states, which are the QC core.

Here we show the operation of a quantum transmitter, Alice, simulated by CCRs of an orbiting satellite and the discrimination of different polarization states by Bob, the receiver on the ground. As sketched in Fig. 1, Alice qubit stream is realized from a $100 \mathrm{MHz}$ train of pulses sent upward from the ground and reflected by the CCRs. After

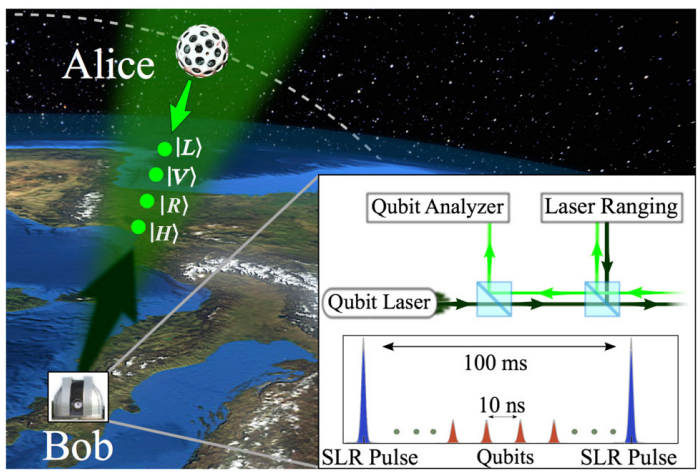

FIG. 1 (color online). Scheme of the satellite QKD demonstration. Qubit pulses are sent at a $100 \mathrm{MHz}$ repetition rate and are reflected back to the single photon level from the satellite, thus mimicking a QKD source in space. Synchronization was performed by using the bright SLR pulses at a repetition rate of $10 \mathrm{~Hz}$. 
the assessment of the uplink attenuation, we set the pulse energy such that the pulses reflected from the satellites have an average photon number $\mu_{\text {sat }}$ close to one. At Bob, located at the Matera Laser Ranging Observatory (MLRO) of the Italian Space Agency in Matera, Italy, we measured an average quantum bit error ratio (QBER) typically lower than $5 \%$. We thus proved the feasibility of the BB84 protocol [23] from satellite to ground with the qubits encoded in four different polarization states of two mutually unbiased bases.

Setup.-In order to minimize the background radiation and dark counts, it is of paramount importance, on the Bob side, to minimize the dwell time of the qubit detection: the latter is related to the precision in the assessment of the expected qubit times of arrivals $t_{\text {ref. }}$. For this purpose, the qubit stream was synchronized to the intense $10 \mathrm{~Hz}$ satellite laser ranging (SLR) pulse train. In this way, $t_{\text {ref }}$ may be precisely derived from the return signal of the SLR. More in detail, a mode-locked laser (a master oscillator) is used as an event generator for the SLR and qubit pulses, imposing an intrinsic synchronization of the two signals. The master oscillator produces pulses at $1064 \mathrm{~ns}, 100 \mathrm{ps}$ duration, and $100 \mathrm{MHz}$ repetition rate synchronized with the MLRO atomic clock. The beam is split to provide the seed for the SLR signal and for the qubit pulses. The high energy SLR signal is obtained by selecting one pulse every $10^{7}$ with a pulse picker and then using three amplifiers and a second harmonic generation (SHG) stage, obtaining pulses at a $532 \mathrm{~nm}, 100 \mathrm{~mJ}$ energy, and $10 \mathrm{~Hz}$ repetition rate. The qubits are obtained with a suitable SHG unit pumped by the remainder of the master oscillator beam at $100 \mathrm{MHz}$, resulting in a train of pulses with an energy of $1.1 \mathrm{~nJ}$. Because of the MLRO atomic clock control on the master oscillator, we achieved a $10 \mathrm{~ns}$ separation of the qubit pulses and the precise time relation with the SLR pulses; see Fig. 1. Two nonpolarizing beam splitters (BSs) are used to combine the SLR and the qubit pulse train in the upward beam that is directed via the Coude path to the MLRO telescope, from which it propagates toward the satellite (more details are provided in the Supplemental Material [24]). The beam divergence is controlled by a collimator, while the polarization state is changed by two wave plates and a modulator.

The qubit stream coming from satellites is collected by the MLRO telescope, propagates along the Coudé path, and is directed to the state measurement by the BSs also used in the uplink. The qubit state analyzer is composed by a rotating wave plate, an optical shutter, and two single photon photomultipliers (PMTs) (Hamamatsu H7360-02, dark counts $<50$ cps, detection efficiency $\eta \sim 10 \%, 22 \mathrm{~mm}$ diameter) placed at the outputs of a polarizing beam splitter. The signals detected by the PMTs are fed into a time tagger with 81 ps resolution. The rotating wave plate, controlled by software, is used to change between two receiving bases, $\{|H\rangle,|V\rangle\}$ and $\{|L\rangle,|R\rangle\}$. For qubit discrimination, we synchronized the state analyzer with the SLR start and stop signals, which are generated by MLRO electronics with picosecond accuracy. By dividing the intervals between two consecutive SLR detections in $10^{7}$ equidistant subintervals, we determined the sequence of expected $t_{\text {ref }}$. This technique automatically compensates for the variation of the round-trip time duration due to the satellite motion. Our detection accuracy $\sigma$ was set equal to the detector timing jitter $(0.5 \mathrm{~ns})$, as other contributions to time uncertainties coming from detection electronics or laser fluctuations are negligible. To evaluate the QBER, counts registered within a $\pm 0.5 \mathrm{~ns}$ (corresponding to $\pm 1 \sigma$ ) interval around $t_{\text {ref }}$ were considered as the signal, while the background has been estimated from the counts outside $\pm 3 \mathrm{~ns}(6 \sigma)$. A significant fluorescence is emitted by the BSs irradiated by the outgoing beam, causing an increase in the background noise. To mitigate such an effect, separate transmission and measurement phases were implemented by means of two mechanical shutters. In the first half of the $100 \mathrm{~ms}$ slot between two SLR pulses, the transmitter shutter is opened while the receiver one is closed, to protect the receiver PMTs. In the second half of the slot the shutter controls are reversed and the detectors receive the qubits from the satellite. With this protocol, the effective transmission time during a slot cannot be larger than the round-trip time; however, since our shutters require about $2 \mathrm{~ms}$ to fully open and $2.5 \mathrm{~ms}$ to fully close, the effective period is restricted by $4.5 \mathrm{~ms}$.

Experimental results.-CCRs with metallic coating on the three reflecting faces are essential for preserving the polarization state during the reflection, as required for the polarization encoding. We selected four low Earth orbit (LEO) satellites with such polarization preserving CCRs as Jason-2, Larets, Starlette, and Stella and, for comparison, one satellite with uncoated CCRs, Ajisai.

QC using generic polarization states from two mutually unbiased bases was first realized with a single passage of Larets, whose passage was divided into four intervals of $10 \mathrm{~s}$ in which we sent horizontal $|H\rangle$, vertical $|V\rangle$, left circular $|L\rangle$ and right circular $|R\rangle$ states. At the receiver, we used two single photon detectors measuring two orthogonal polarizations, from which the QBER is extracted. The QBER was estimated as $Q=\left(n_{\text {wrong }}+1\right) /\left(n_{\text {corr }}+\right.$ $\left.n_{\text {wrong }}+2\right)$ where $n_{\text {corr }}$ and $n_{\text {wrong }}$ are the number of detections in the transmitted and orthogonal polarizations, respectively (we used the Bayesian estimator of the QBER; see the Supplemental Material [24]). As an example, the passage starting at 02:40 universal time of April 10, 2014, provided the results shown in Fig. 2. In the four intervals, we obtained 199 counts in the correct detector and 13 wrong counts, giving an average QBER of $6.5 \% \pm 1.7 \%$, which is suitable for secret key extraction. Considering the average $3.6 \%$ duty cycle of our setup, the mean return rate in the selected intervals is $147 \pm 10 \mathrm{cps}$. This rate corresponds to $\sim 10^{4}$ bits for each Larets passage in the case of clean sky conditions when using fast shutters. We note that 


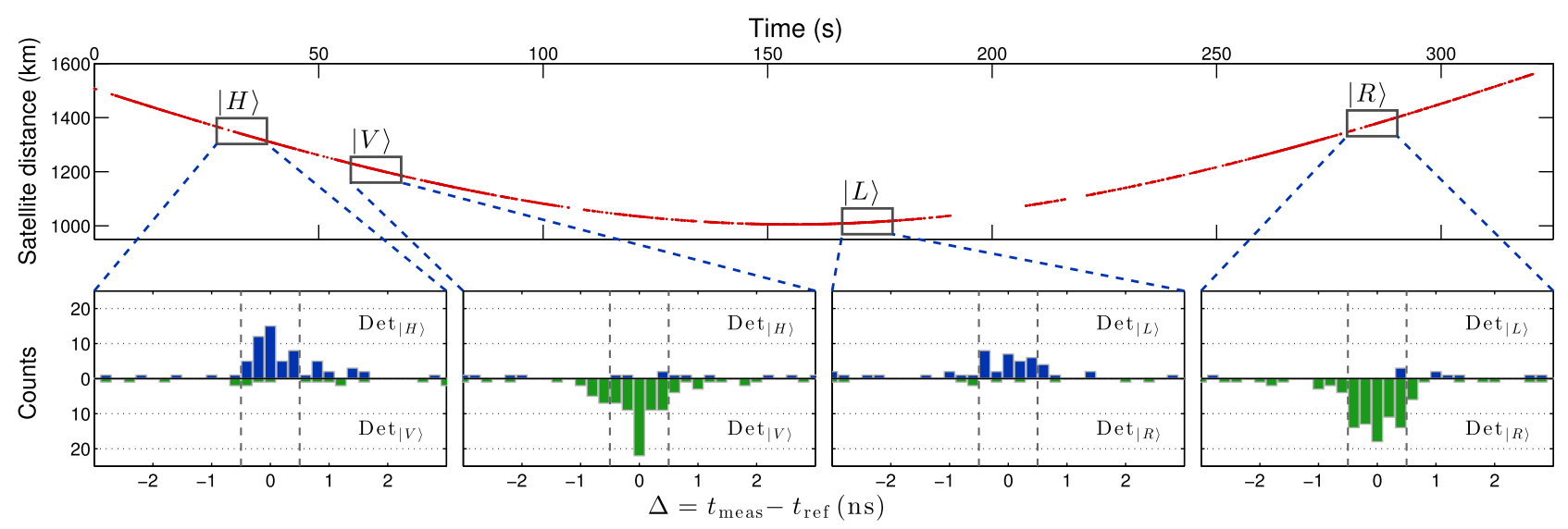

FIG. 2 (color online). Qubits return. (Top panel) Larets trajectory measured by the $10 \mathrm{~Hz}$ SLR pulses. The four selected $10 \mathrm{~s}$ intervals correspond to four different polarization input states. (Bottom panel) The four histograms report the obtained counts at the receiver for each single photon detector in a function of the measured detection time $t_{\text {meas }}$, demonstrating an average estimated QBER of $6.5 \% \pm 1.7 \%$. The signal on the two detectors is blue for $H / L$ polarization and green for $V / R$. Vertical gray dashed lines represent the $1 \sigma$ selection interval around the expected time of arrival $t_{\text {ref }}$. Lower counts in the $|L\rangle$ state are due to a lower duty cycle caused by the shorter distance of the satellite compared to the other three states.

the duty cycle of our scheme may be increased up to $50 \%$ by using faster shutters.

A further analysis has been carried out to demonstrate the feasibility of polarization encoding with other satellites as well. In this analysis, we divided the detection period in $5 \mathrm{~s}$ intervals that were then analyzed only if the signal resulted in 5 standard deviations above the background from at least one detector. The QBERs resulting from this analysis are shown in Fig. 3. We achieved low QBER for several tens of seconds in all of the polarization maintaining satellites (Jason-2, Larets, Starlette, and Stella), with an average value for each passage not exceeding $7 \%$ (the Larets results shown in Fig. 3 refer to a different passage with respect to Fig. 2). Lower QBER was measured for the relatively new satellites Larets and Jason-2 (launched, respectively, in 2003 and 2008). Stella and Starlette were launched in 1993 and 1976 and their larger QBER could be explained by a wearing of the reflection coating. As expected, for Ajisai, having CCRs non preserving the polarization state, the QBER is above $40 \%$. By combining together the results of all of the polarization maintaining satellites we achieved an overall communication period of $85 \mathrm{~s}$, with an average QBER $=4.6 \% \pm 0.8 \%$. In Fig. 3 we also report the experimental detection rates $(f)$ achieved with the different LEO satellites. These results prove that faithful transmission of different polarization qubits can be obtained in different conditions and satellite orbits. They also show the stability and the reliability of our approach, demonstrating the feasibility of the BB84 protocol [23] from satellite to ground, which requires an average QBER below $11 \%$ [25].

Estimation of $\mu_{\text {sat }}$-The implementation of Earthsatellite QKD makes use of faint laser pulses with a mean photon number of the Poisson process $\mu$ close to 1 . Indeed, the BB84 protocol with decoy states [27] in a realistic scenario requires $\mu \lesssim 2[28]$ and decoy signals with a mean photon number close to 1 [29] (see also the Supplemental Material [24]). The estimation of the average number of photons per pulse leaving the satellites in all directions, $\mu_{\text {sat }}$, is obtained in our experiment by dividing the average number of photons per pulse detected at the receiver, $\mu_{\mathrm{rx}}$, by

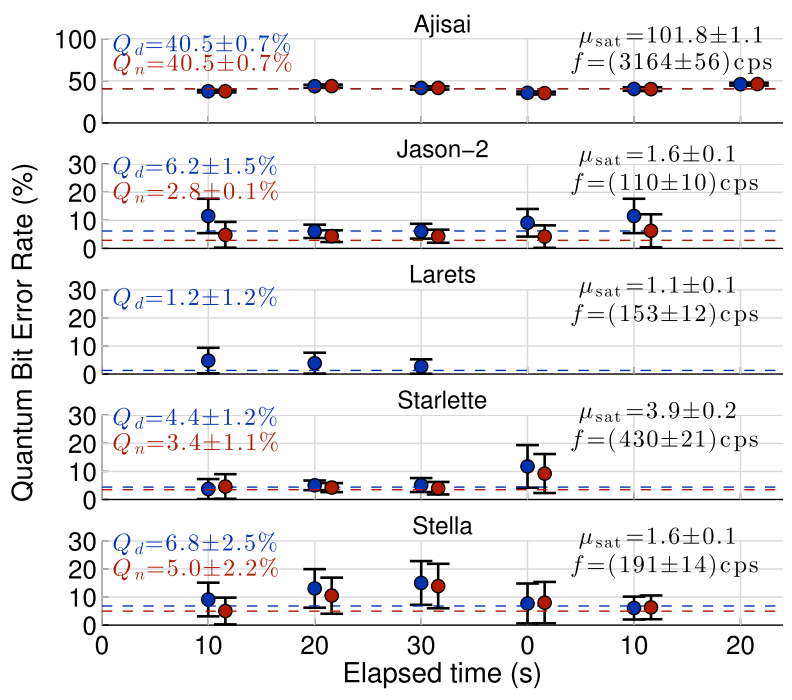

FIG. 3 (color online). QBER of the received signals. We fixed the sent polarization to $|V\rangle$ and measured in two orthogonal polarizations, $|H\rangle$ and $|V\rangle$. For each satellite we show the bare QBER (blue dots) and the QBER calculated after the background subtraction (red dots). Error bars represent Poissonian errors. Dashed lines represent $Q_{d}$ and $Q_{n}$, the bare and background subtracted QBER for the whole satellite acquisition. For Larets we observed no detection in the wrong state, and we did not estimate the QBER with background subtraction. The coating of Ajisai retroreflectors depolarizes the qubits, while the other satellites preserve the photon polarization. We also indicate the mean detection rate and the average photon number per pulse at the satellite. 
the transmittivity of the quantum channel. A general formula to predict the detected number of photons per pulse is the radar equation [30]:

$$
\mu_{\mathrm{rx}}=\mu_{\mathrm{tx}} \eta_{\mathrm{tx}} G_{t} \Sigma\left(\frac{1}{4 \pi R^{2}}\right)^{2} T_{a}^{2} A_{t} \eta_{\mathrm{rx}} \eta_{\mathrm{det}}
$$

where $\mu_{\mathrm{tx}}$ is the source mean photon per pulse, $\eta_{\mathrm{tx}}$ is the optical transmission efficiency, $G_{t}$ is the transmission gain, $\Sigma$ and $R$ are the satellite cross section and slant distance, $T_{a}$ is the atmospheric transmissivity, $A_{t}$ is the telescope area, $\eta_{\mathrm{rx}}$ is the optical receiving efficiency, and $\eta_{\operatorname{det}}$ is the single photon detector efficiency [30]. To estimate $\mu_{\text {sat }}$ it is necessary to factorize Eq. (1) into uplink and downlink contributions. While most parameters may be easily ascribed to uplink or downlink, the satellite cross section $\Sigma=\rho A_{\text {eff }} G_{\text {down }}$ combines terms of both and has to be split. The parameters $\rho$ and $A_{\text {eff }}$, corresponding to the CCR reflectivity and the effective satellite retroreflective area, contribute to the uplink, while $G_{\text {down }}$ expresses the effective downlink gain. The downlink factor is $G_{\text {down }}\left(1 / 4 \pi R^{2}\right) T_{a} A_{t} \eta_{\mathrm{rx}} \eta_{\text {det }}$ and was used to estimate $\mu_{\text {sat }}$ as

$$
\mu_{\mathrm{rx}}=\mu_{\mathrm{sat}} G_{\mathrm{down}}\left(\frac{1}{4 \pi R^{2}}\right) T_{a} A_{t} \eta_{\mathrm{rx}} \eta_{\mathrm{det}} .
$$

The values of the parameters used to evaluate $\mu_{\text {sat }}$ are listed in the Supplemental Material [24]. In particular, for the satellite cross sections, we used those given in Ref. [31-33]. For the Larets passage of Fig. 2, we obtained $\mu_{\text {sat }}$ 's equal to $0.9,1.0$, 0.6 , and 1.2 for the $|H\rangle,|V\rangle,|L\rangle$, and $|R\rangle$ polarizations, respectively (the uncertainty is 0.1 in all of the cases). These values correspond to an average $\mu_{\text {sat }}$ of $0.9 \pm 0.1$ and a corresponding average downlink transmissivity of $\sim 2.4 \times 10^{-6}(56 \mathrm{~dB})$. The values of $\mu_{\text {sat }}$ for the remaining satellites are reported in Fig. 3. The resulting $\mu_{\text {sat }}$ is on the order of unity for the four satellites with metallic CCRs.

The full radar equation has been used to extrapolate the transmitter gain, given by

$$
G_{t}=\frac{8}{\theta_{t}^{2}} \exp \left[-2\left(\frac{\theta}{\theta_{t}}\right)^{2}\right] .
$$

In the previous equation, $\theta_{t}$ is the divergence angle of the upgoing beam (including beam broadening due to turbulence), while $\theta$ is the pointing error. Since the two parameters $\theta$ and $\theta_{t}$ cannot be directly and separately measured, we obtained an estimate for $G_{t}$ by averaging the data obtained in different passages of the several LEO satellites. Because of pointing fluctuations, the qubit detection frequency also fluctuates during the passages, typically on a scale of seconds. Accordingly, we divided the measurement into $10 \mathrm{~s}$ periods and selected those with better SNR. Therefore, from the most stable data taken for Ajisai, Jason, and Starlette, we estimated $G_{t}=4.1 \times 10^{8}$. The latter has been used in the radar equation to predict the number of received photons, which are compared in Fig. 4

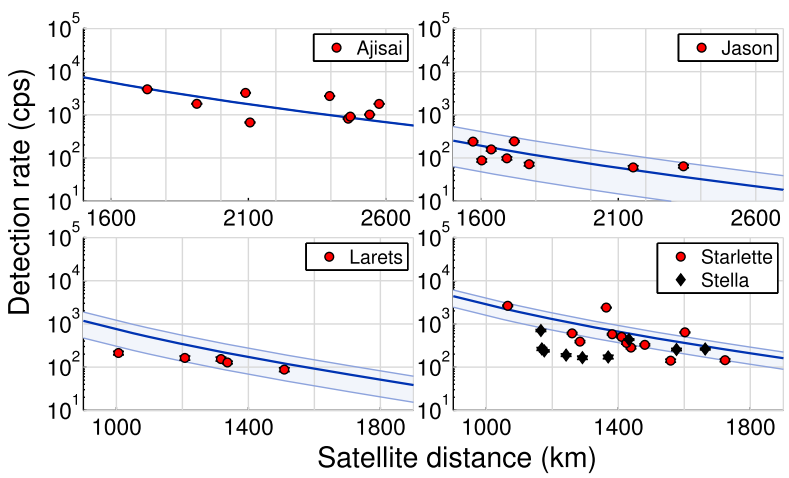

FIG. 4 (color online). Detection rates and link budgets. Points represent the return detection rates of the qubits for different satellites along the orbit, compared with the prediction of the link budget provided by Eq. (1) (the continuous line). Error bars account for Poissonian errors only, while the shaded area comes from the available uncertainties of the satellite cross sections $\Sigma$. Uncertainties in the orbital parameters and beam pointing affect the trend of the return rate beyond the shot noise. The twin satellites Stella and Starlette show different behavior despite similar characteristics, but in line with the SLR statistics that could be explained by a wearing of the Stella CCR coating.

with the measured ones. Here we demonstrate that the radar equation model [30] and Eq. (2) provides a precise fit for the measured counts and the $\mu_{\text {sat }}$ values shown in Fig. 3. It is worth noticing that the same value, $G_{t}=4.1 \times 10^{8}$, was used to predict all of the link budgets shown in Fig. 4 and that this corresponds to a consistency check of our $\mu_{\text {sat }}$ estimation.

Two-way protocol.-The QC scheme here described could be exploited as a practical satellite QKD system. Indeed, as both beams share the same path, we note that the polarization transformation induced by the telescope movements in the uplink is compensated for in the downlink (see the Supplemental Material [24] for the explicit derivation). By introducing a state transformation during the retroreflection at the satellite, controlled by Alice, it is possible to encode the desired state to be sent to Bob on the ground. The protocol works as follows: at the ground station, a horizontally polarized train of pulses is prepared and sent via the Coudé path to the telescope. The exit polarization is rotated by a given angle, depending on the telescope pointing direction. The pulses are directed toward a satellite equipped with a polarization rotator in front of the CCRssuch as a Faraday rotator or more advanced devices, as reported in Ref. [34] — which imposes to the returning qubit a suitable polarization rotation $\phi$. The measure of the irradiance at the satellite will also be realized, to avoid a Trojan horse attack [35] and then to ensure the security of the protocol. In addition, a suitable attenuator is used to assess the desired $\mu_{\text {sat }}$. The retroreflected beam then propagates toward the ground telescope, and thanks to the properties of the Coudé path, a polarization rotated by $\phi$ with respect to the horizontal will be received. By this 
scheme, a decoy state BB84 protocol can be realized between the satellite and the ground exploiting the precise pointing provided by the CCR and enjoying a very compact payload. The experimental results shown above demonstrate that such a protocol is currently realizable using CCRs of a few centimeters and that the MLRO is suitable for space QC.

Conclusions.-We experimentally demonstrated the preservation of single photon polarization over a satellite-to-ground channel, covering an unprecedented length compared to ground experiments. We showed the experimental realization of QC from several satellites acting as a quantum transmitter and with the MLRO as the receiver. QBER was found to be low enough to demonstrate the feasibility of quantum information protocols such as QKD along a space channel. Moreover, we propose that a very simple trusted device in orbit, formed by an active CCR mounted on a spacecraft and operated in the two-way scheme, may provide a simple alternative to a space terminal based on conventional QKD design. Since all existing SLR facilities can be turned into QC stations with a minor upgrade, our results foster a faster expansion of QC around the planet and beyond.

We would like to thank Francesco Schiavone, Giuseppe Nicoletti, and the MRLO technical operators and Prof. Cesare Barbieri, Dr. Andrea Tomaello, and Dr. Alberto Dall'Arche (of the University of Padova) for the collaboration and support. We also thank Prof. A. Sokolov for giving us useful information about the CCR of the Larets satellite. This work has been carried out within the Strategic-Research-Project QUANTUMFUTURE (STPD08ZXSJ) of the University of Padova and the Strategic-Research-Project QUINTET of the Department of Information Engineering, University of Padova.

"paolo.villoresi@dei.unipd.it

[1] C. H. Bennett and P. W. Shor, Science 284, 747 (1999).

[2] H. Lo and H. F. Chau, Science 283, 2050 (1999).

[3] R. Hughes and J. Nordholt, Science 333, 1584 (2011).

[4] D. Bacco, M. Canale, N. Laurenti, G. Vallone, and P. Villoresi, Nat. Commun. 4, 2363 (2013).

[5] S. Etcheverry, G. Cañas, E. S. Gómez, W. A. T. Nogueira, C. Saavedra, G. B. Xavier, and G. Lima, Sci. Rep. 3, 2316 (2013).

[6] M. Mirhosseini, O. S. Magaña-Loaiza, M. N. O'Sullivan, B. Rodenburg, M. P. J. Lavery, M. J. Padgett, D. J. Gauthier, and R. W. Boyd, New J. Phys. 17, 033033 (2015).

[7] M. Peev et al., New J. Phys. 11, 075001 (2009).

[8] M. Sasaki et al., Opt. Express 19, 10387 (2011).

[9] B. Fröhlich, J. F. Dynes, M. Lucamarini, A. W. Sharpe, Z. Yuan, and A. J. Shields, Nature (London) 501, 69 (2013).

[10] X.-S. Ma et al., Nature (London) 489, 269 (2012).

[11] H. Xin, Science 332, 904 (2011).

[12] Quantum Information and Communication European roadmap: http://qurope.eu/projects/quie2t/wp2/deliverables; Roadmap of Quantum ICT Laboratory of National Institute of Information and Communications Technology of Japan: http://www.nict.go.jp/en/advanced_ict/quantum/roadmap .html; Quantum Information Science and Technology Roadmap of USA: http://qist.lanl.gov/qcomp_map.shtml.

[13] M. Żukowski, A. Zeilinger, M. A. Horne, and A. K. Ekert, Phys. Rev. Lett. 71, 4287 (1993).

[14] A. J. Leggett, J. Phys. Condens. Matter 14, R415 (2002).

[15] D. Rideout et al., Classical Quantum Gravity 29, 224011 (2012).

[16] M. Aspelmeyer, T. Jennewein, M. Pfennigbauer, W. Leeb, and A. Zeilinger, IEEE J. Sel. Top. Quantum Electron. 9, 1541 (2003).

[17] C. Bonato, M. Aspelmeyer, T. Jennewein, C. Pernechele, P. Villoresi, and A. Zeilinger, Opt. Express 14, 10050 (2006).

[18] A. Tomaello, C. Bonato, V. Da Deppo, G. Naletto, and P. Villoresi, Adv. Space Res. 47, 802 (2011).

[19] J.-P. Bourgoin et al., New J. Phys. 15, 023006 (2013).

[20] T. Scheidl, E. Wille, and R. Ursin, New J. Phys. 15, 043008 (2013).

[21] P. Villoresi et al., New J. Phys. 10, 033038 (2008).

[22] J. Yin, Y. Cao, S.-B. Liu, G.-S. Pan, T. Yang, Z.-P. Zhang, F.-M. Yang, Y.-A. Chen, C.-Z. Peng, and J.-W. Pan, Opt. Express 21, 20032 (2013).

[23] C. H. Bennett and G. Brassard, in Proceedings of the IEEE International Conference on Computers, Systems and Signal Processing, Bangalore, India (IEEE, New York, 1984), p. 175.

[24] See Supplemental Material at http://link.aps.org/ supplemental/10.1103/PhysRevLett.115.040502 for details of the experimental implementation and of the results. A detailed discussion on the three decoy state protocol is also presented.

[25] By using the postselection techniques introduced in Ref. [26], QBER up to $15 \%$ can be tolerated for (reasonable) secret key generation.

[26] J. Bae and A. Acín, Phys. Rev. A 75, 012334 (2007).

[27] X. Ma, B. Qi, Y. Zhao, and H.-K. Lo, Phys. Rev. A 72, 012326 (2005).

[28] V. Scarani, H. Bechmann-Pasquinucci, N. Cerf, M. Dušek, N. Lütkenhaus, and M. Peev, Rev. Mod. Phys. 81, 1301 (2009).

[29] T. Tsurumaru, A. Soujaeff, and S. Takeuchi, Phys. Rev. A 77, 022319 (2008).

[30] J. Degnan, Geodyn. Ser. 25, 133 (1993).

[31] D. A. Arnold, Cross section of ILRS satellites, report, 2003, http://ilrs.gsfc.nasa.gov/docs/2003/CrossSectionReport.pdf.

[32] A. L. Sokolov (private communication); V. P. Vasilev and I. S. Gashkin, Improved ball-lens retroreflector satellite for operation in higher orbits, 2012, http://www.ipa.nw.ru/ conference/wpltn2012/docs/25/1500\%20sokolov.pdf (unpublished).

[33] V. B. Burmistrov, N. N. Parkhomenko, V. D. Shargorodsky, and V. P. Vasiliev, in Proceedings of the 14th International Workshop on Laser Ranging, San Fernando, Spain, 2004, http://cddis.nasa.gov/lw13/docs/presentations/ target_parkhomenko_1p.pdf (unpublished).

[34] I. Lucio-Martinez, P. Chan, X. Mo, S. Hosier, and W. Tittel, New J. Phys. 11, 095001 (2009).

[35] N. Gisin, S. Fasel, B. Kraus, H. Zbinden, and G. Ribordy, Phys. Rev. A 73, 022320 (2006). 